\title{
Embedding an allied health service in the Nelson Hospital Emergency Department: A retrospective report of a six month pilot project
}

Clare Holmes Dip Phty

Physiotherapist, Emergency Department, Nelson Hospital, Nelson Marlborough District Health Board

Debbie Hollebon Grad Dip Soc Wk, Reg Soc Wk, MANZASW

Social Worker, Emergency Department, Nelson Hospital, Nelson Marlborough District Health Board

Alice Scranney BSC (Hons), BPhty

Physiotherapist, Emergency Department, Nelson Hospital, Nelson Marlborough District Health Board

Hilary Exton Grad Dip Phys, BSc (Hons), BPhty

Director and Service Manager Allied Health, Nelson Hospital, Nelson Marlborough District Health Board

\section{ABSTRACT}

Embedding allied health into the emergency department (ED) was considered the best option by a 2011 Nelson Marlborough District Health Board (NMDHB) strategic planning group exploring innovative ways to bridge the gap between primary care and the ED and thus enhance the service. A six month pilot project implemented in 2012 aimed to provide a more timely multidisciplinary approach to patient care, facilitate safe discharge and linkages into the primary care sector, reduce multiple presentations and keep within ED designated time frames. Physiotherapy and social work practitioners worked as part of an inter-disciplinary team to comprehensively assess clients' specific needs. The establishment of primary physiotherapy contact, where appropriate patients were identified directly from initial presentation, solely assessed and treated by the physiotherapist improved patient flow for lower triage presentations and made full use of staffing resources. Data collection over the first 22 weeks showed the allied health practitioners in ED had 749 new patient contacts, including 120 primary physiotherapy contacts. ED patients found the service extremely helpful. Survey responses from key stakeholders and ED staff highlighted the benefit of allied health interventions in improving safety of ED discharges, and the value of comprehensive client centred patient care. The Executive Leadership Team of the NMDHB unanimously endorsed the continuation of the allied health service in the emergency department from December 2012.

Holmes C, Hollebon D, Scranney A, Exton H (2016) Embedding an Allied Health Service in the Nelson Hospital Emergency Department: a retrospective report of a six month pilot project. New Zealand Journal of Physiotherapy 44(1): 17-25. doi: 10.15619/NZJP/44.1.03

Key Words: Physiotherapy, Social Work, Emergency Department, Allied Health, Multidisciplinary team

$\ldots \ldots \ldots \ldots \ldots \ldots \ldots \ldots \ldots \ldots \ldots \ldots \ldots \ldots \ldots \ldots \ldots \ldots \ldots \ldots \ldots \ldots \ldots \ldots \ldots \ldots \ldots \ldots \ldots \ldots \ldots \ldots \ldots \ldots \ldots \ldots \ldots \ldots \ldots \ldots \ldots \ldots$

\section{INTRODUCTION}

Changing population demographics with an increase in the number of people presenting with often multiple long term conditions have placed strain on emergency department (ED) service provision in New Zealand and internationally. The traditional medical ED model of care is considered not well suited to fully manage the comprehensive package of care these patients require (Rea et al 2010). Also, there are increasing issues in providing the care required within expected time frames. Different models of ED care (Australian and New Zealand Society for Geriatric Medicine 2008, Ministry of Health 2008, 2011, New South Wales Health 2006, The National Ageing Research Institute Australia 2007) point to improved access to allied health as being important in care provision.

Limited studies have been undertaken in the UK (Jibuike et al 2003, McClellan et al 2005, 2010), Australia (Farrell 2014, Gill and Stella 2013, Lau et al 2008, Morris et al 2015) and New Zealand (Canterbury District Health Board 2006) on the introduction and benefits to ED of physiotherapy. These studies largely looked at the role of an ED physiotherapist in an advanced scope of practice (primary contact) role treating musculoskeletal presentations or specific injuries. Patient satisfaction was high, with decreased wait times for more minor injuries shown. However, there were no major differences in long term patient outcomes. Physiotherapy was felt to be a beneficial adjunct to ED care, especially in reduction of acute musculoskeletal back pain (Lau et al 2008). Two studies explored and discussed the positive impact the social work ED role could make to emergency care and costs by addressing client psychosocial issues. These studies speak positively but have limited evidence (Bywaters et al 2003, Van Pelt 2010). Studies looking at a multidisciplinary team approach to ED patient care in relation to high intensity users and complex presentations showed stronger links with the primary care sector and improved discharge planning (Moss et al 2002, Rea et al 2010).

In 2011, the Director of Allied Health and the Clinical Director of the Emergency Department Nelson became the sponsors of a project to best manage the identified gap in service delivery between the ED and primary sector (Ministry of Health 2011). The gap was caused by the increasing complexity and multi 
factorial nature of presentations. The previous system of paging allied health services from elsewhere in the hospital did not provide a timely coordinated service. Due to time, bed space constraints, and ED staff not being fully aware of the benefits of allied health input, complex patients often would not receive allied health assessment and interventions.

The aims of the pilot project were to: (1) provide comprehensive patient care by a multidisciplinary team (MDT) in a timely manner, ensuring patient allied health needs were fully addressed, (2) support and enhance the ED six hours length of stay target required by the Ministry of Health (Ministry of Health 2011), and (3) facilitate safe discharge and linkages into the primary care sector, address falls risk issues, reduce multiple presentations and create management plans where appropriate.

This paper describes the implementation and delivery of a physiotherapy and social work service in ED and investigates patient, staff and key stakeholder perceptions of allied health input in ED.

\section{METHOD}

Nelson Emergency Department (ED) serves a catchment of over 100,000 people in the Nelson Region, providing a 24 hour service, seven days a week. It is categorised as a Level four dedicated 17 bed unit. The ED service sees approximately 25,000 patients per annum, seeing patients with serious injury or illness as a priority using the Australasian Triage System (ATS) with triage guideline categories one to five. Triage 1 patients are deemed medical emergencies requiring medical assessment and management immediately. Medically unstable patients should receive care within 10 minutes (triage 2 ) or medically unwell, within 30 minutes (triage 3). Triage 4 and 5 patients are deemed medically stable suffering non-life threatening injuries requiring input within 1 hour (triage 4) or 2 hours (triage 5).

In 2009, a weeklong trial of allied health service within ED in Nelson was undertaken. This short trial was valuable in deciding the parameters and direction of the 2012 planned pilot project, and also ensured the engagement of the whole ED team. Two allied health professions were chosen to be trialled in the pilot project: social work and physiotherapy. It was considered that these two professions could provide a wide skill base in practical assessment and management of physical, mobility, rehabilitation, support and psychosocial issues, and could link with other allied health services as required. Utilising the New Zealand legislation for direct physiotherapy referral (HPCA 2003), the pilot project established primary physiotherapy contact, where suitable patients were assessed and managed solely by the physiotherapist direct from the initial triage nurse assessment.
A six month pilot project to deliver both social work and physiotherapy services from within the ED was implemented in 2012. A working group between allied health, medical, nursing and key stakeholders ensured a robust quality, safety and clinical governance framework was in place. Due to the pilot process, time was set aside for regular meetings and initiatives related to the pilot.

Stakeholders involved in the pilot included Service Directors, Clinical Leaders for Allied Health Medical and Nursing, Support Works, Service Managers for Allied Health and Medical Directorate, Violence Intervention Co-ordinator, Nga Pukenga Hauora (an inpatient Māori Health support service), Paediatric Service, Alcohol and Drugs Service, Mental Health Services, Nelson Bays Primary Health Organisation, Primary Care (medical practitioners), community health providers, Accident Compensation Corporation, and the Medical and Injury Centre.

Staff Recruitment and Training

Clinicians with a minimum of five years post-graduate experience were recruited to fill the roles of full time social worker and full time physiotherapist. The allied health team consisted of three staff, the physiotherapy role being filled in a job share arrangement. Staff underwent a training and orientation programme in the ED and relevant information technology systems. Physiotherapists became accredited to issue basic occupational therapy equipment, and reviewed orthotic options. While the pilot was underway the physiotherapists underwent the Nelson Hospital X-ray training to be aligned to the established Nurse Initiated X-ray (NIXR) protocol for $X$-ray ordering, also plastering and splint workshops for simple fracture management. The social worker spent time in the community liaising with primary care services and community organisations to develop a strong knowledge of available options for clients.

\section{Patient Referral and Selection Criteria}

All patients presenting to ED were initially triaged by a nurse. A primary physiotherapy contact framework with clear inclusion and exclusion criteria was developed by physiotherapy personnel to provide safety parameters for appropriate referral. Patients to be recruited to the service were triage 4 and 5 patients with primarily musculoskeletal injuries. Exclusion criteria for primary physiotherapy intervention were patients presenting with constant unremitting pain, cauda equina signs and symptoms, raised temperature or signs of infection, significant mechanism of action/head injury, open wounds, children under five years, major deformities, confusion or altered level of consciousness, recent seizure activity, marked neurological change, and observations triggering an Early Warning Score (a system to detect abnormal observations of vital signs). 


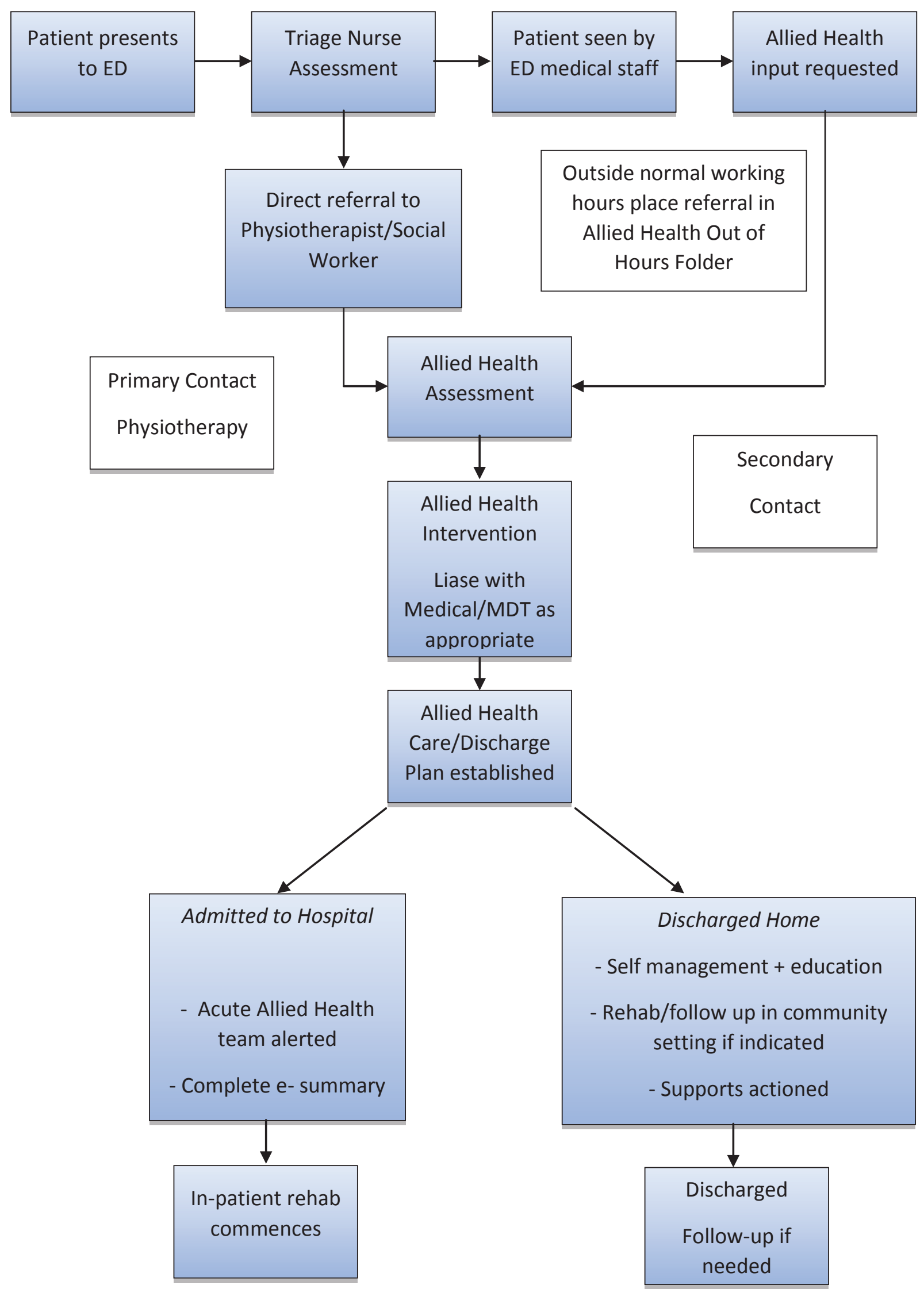

Figure 1: Emergency Department allied health intervention flowchart 
Figure 1 shows the patient pathway through ED when allied health intervention was indicated. Physiotherapists were able to access primary referrals directly following the triage process either by verbal contact from the triage nurse or reviewing the patient folder. The triage nurse could also refer directly to the social worker if appropriate, who would then liaise with the primary clinician. Secondary referrals for both social work and physiotherapy were usually by direct verbal contact or phone, easily enabled by being embedded in the department. Physiotherapists proactively targeted any patients aged over 65 years presenting following a fall, or with a high falls risk. Following care, patients returned to community and primary care services or were admitted to hospital.

Multiple presenters are patients who present frequently to ED over a short time period with complaints not always appropriate for an emergency service that usually could be well managed in the primary sector. A group initiative including the ED social worker, ED team leaders, Registered Nurse Multiple Presenter coordinator, Mental Health representative, and a representative from St John (a charitable organisation providing frontline medical response) met regularly in 2012. The group's aim was to focus on the most frequent multiple presenters, to identify their main issues and see if or how their needs could be best met in the primary sector. The social worker had an active role in this process, linking patients with appropriate primary care services, facilitating multidisciplinary team meetings and helping develop workable management plans.

\section{Data Collection and Analysis}

As an audit of the new service, a data collection system and agreed evaluation framework were developed. Data were initially collected over a 22 week period from 30 April till 1 October 2012 for presentation to the Executive Leadership Team. A further data collection was undertaken at 35 weeks, the week of 31 December 2012. The allied health team recorded all allied health contacts, either new or follow-up, documenting the time seen, intervention reason, duration of intervention, onward referral pathway, and other patient related activity. Non-patient related activity was also captured. To assess benefits of allied health input outside normal working hours, practitioners worked several later shifts into the evenings and weekend days during the pilot. An "Allied Health After Hours"

\section{Table 1: Number of allied health contacts}

\begin{tabular}{|c|c|c|c|}
\hline Discipline & Patient Contact & Week 1 - Week 22 & Week 1 - Week 35 \\
\hline \multirow[t]{4}{*}{ Physiotherapy } & New Patients & 429 & 824 \\
\hline & (Primary Contacts) & $(120)$ & $(258)$ \\
\hline & Follow ups & 45 & 50 \\
\hline & Total Contacts & 474 & 874 \\
\hline \multirow[t]{3}{*}{ Social Work } & New Patients & 320 & 510 \\
\hline & Follow Ups & 287 & 459 \\
\hline & Total Contacts & 607 & 969 \\
\hline \multirow[t]{2}{*}{ Total Allied Health contacts in ED } & New Patients & 749 & 1334 \\
\hline & Total Contacts & 1081 & 1843 \\
\hline
\end{tabular}

Notes: ED, Emergency Department

folder was made available for ED staff to alert allied health to patients presenting outside normal work hours. These patients were followed up the next working day, and interventions instigated as appropriate.

Five months after initiation of the pilot project written questionnaires, especially designed for the project, were offered to ED patients, ED staff and stakeholders (as listed above) to evaluate how successfully the pilot was progressing. ED patients were asked to rate how helpful the allied health professional was on a scale where 1=extremely helpful and $5=$ unhelpful. Open questions were used to find out in what way patients found the allied health professional helpful and what suggestions they had for improving the service. This selfreport questionnaire was either given to ED patients as they left the department to be completed and deposited in a post box at reception, or posted out and returned by mail. All questionnaires were anonymous.

ED staff received a questionnaire via the internal mail system, and could deposit anonymous replies in a slotted reply box in ED. They were asked to rate the service on a scale of 1 to 5 ( 1 =extremely helpful and $5=$ unhelpful) as to how helpful they believed the allied health service was for ED, then complete open-ended questions on what they valued about the service, what improvements in care and cost savings it provided, the impact of discontinuing the service and ideas for service improvement. Key stakeholders were sent a questionnaire to provide qualitative feedback on how the allied health team had impacted on the wider hospital and primary care facilities, the impact on patients, and financial benefits. Administration staff collated all replies and entered the data into excel spreadsheets.

\section{RESULTS}

The total number of referrals to the allied health professionals represented $7 \%$ of the total ED presentations over the first 22 weeks. Of these $56 \%$ were female, $44 \%$ male, with ethnicity 81.8\% New Zealand European, 10.2\% Māori, 5.9\% European, $1.3 \%$ Asian and $0.7 \%$ Pacific Island or other. There were $2.8 \%$ registered as living outside the NMDHB catchment area, travellers from other areas in New Zealand or overseas. The age range was widespread, with $35 \%$ of allied health contacts being over 65 years, followed by $30 \%$ in the 17 to 44 age group, $18 \%$ in the $45-64$ age group and $17 \% 16$ years and under. 
Table 2: Total allied health non-contact time 22 weeks (\%)

\begin{tabular}{lc}
\hline Non-Contact Activity & Percentage \\
\hline Documentation/Notes/Admin & $28 \%$ \\
Meetings operational & $22 \%$ \\
Conference/study leave/education & $12 \%$ \\
Liaison with Health Professionals & $11 \%$ \\
Meetings clinical (not patient related) & $9 \%$ \\
Presentations/ Teaching & $8 \%$ \\
Liaison Community Organisations & $4 \%$ \\
Travel & $3 \%$ \\
Phone contact (not linked to patient) & $2 \%$ \\
\hline
\end{tabular}

Table 3: Referral reason allied health

\begin{tabular}{lclc}
\hline $\begin{array}{l}\text { Referral Reason } \\
\text { Social Work }\end{array}$ & $\begin{array}{c}\text { New Contacts } \\
\text { 35 weeks }\end{array}$ & $\begin{array}{l}\text { Referral Reason } \\
\text { Physiotherapy }\end{array}$ & $\begin{array}{c}\text { New Contacts } \\
\text { weeks }\end{array}$ \\
\hline Home cares & 186 & MSK Lower limb & 295 \\
Child Concerns & 74 & MSK Upper limb & 186 \\
Family Support & 63 & Falls & 131 \\
Financial & 54 & MSK Spine & 92 \\
Domestic Violence & 41 & Medical/Respiratory & 87 \\
Mental Health & 24 & MSK Multi Body Part & 28 \\
Multiple Presenters & 18 & Other & 5 \\
Alcohol/drugs & 16 & & \\
Loss and Grief & 14 & & \\
Trauma & 10 & & \\
Homeless & 7 & & \\
Isolation & 3 & & \\
\hline
\end{tabular}

Notes: MSK, musculoskeletal

Table 4: Consumer and Emergency Department staff feedback

\begin{tabular}{lccc}
\hline $1-5$ scale of value & $\begin{array}{c}\text { Consumers } \\
\text { Allied Health } \\
(\mathrm{n}=51)\end{array}$ & $\begin{array}{c}\text { ED Staff } \\
\text { Physiotherapy } \\
(\mathrm{n}=42)\end{array}$ & $\begin{array}{c}\text { ED Staff } \\
\text { Social Work } \\
(\mathrm{n}=42)\end{array}$ \\
\hline $1=$ Extremely helpful & 44 & 39 & 41 \\
$2=$ Very helpful & 6 & 3 & 1 \\
$3=$ Helpful & 1 & 0 & 0 \\
$4=$ Not very helpful & 0 & 0 & 0 \\
$5=$ Unhelpful & 0 & 0 & 0 \\
\hline
\end{tabular}

Notes: ED, Emergency Department

Table 1 shows the number of allied health contacts over the first 22 weeks and during the second data collection period from week 1 to week 35. This shows new contacts (including primary contact numbers for physiotherapy in brackets), follow up contacts and total contacts for each profession. Total allied health contacts are listed below. Table 2 presents the nonpatient contact time and the way it was spent during the first 22 weeks of the pilot, documentation and operational meetings taking up most of this time.
The referral reasons for social work and physiotherapy interventions are shown in Table 3. Social work services focused on provision of home care support, child and family issues, addressing multiple presenters and financial stresses. The majority of physiotherapy contacts were for musculoskeletal injuries (MSK) and falls, but there was a diverse spread of presentations for both primary and secondary interventions. 


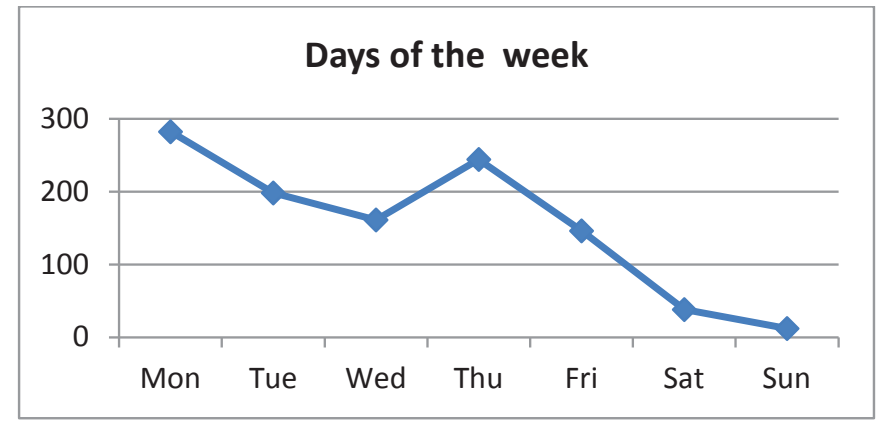

Figure 2: Allied health contacts over days of the week

Figure 2 shows the daily distribution figures of allied health interventions over the first 22 weeks. Presentations over the week were reasonably evenly spread, with an increase on Mondays, largely explained by weekend injuries. Allied health working weekend hours showed higher figures for Saturdays than Sundays, with contact numbers significant considering the few weekends trialled.

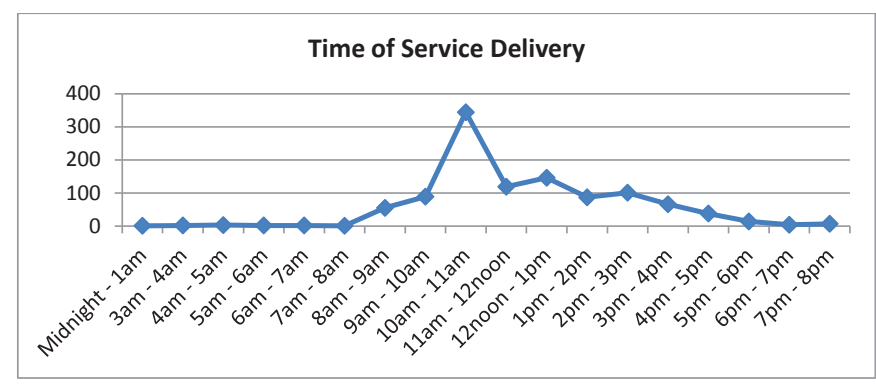

Figure 3: Time of allied health service delivery contacts

Figure 3 shows the daily allied health intervention time over this 22 week period. Intervention times showed a sharp increase in contacts from commencement of work till a peak mid-morning, then a steady flow, tailing off late afternoon/evening. By the end of December 2012 there were 278 referrals recorded in the Allied Health After Hours folder.

Following on from the multiple presenter meetings involving the ED social worker, by the end of 2012, management plans were in place for $70 \%$ of the most frequent ED presenters. These were formulated with client and primary case manager involvement and mutually agreed to.
Onward referrals in the first 22 weeks to community agencies and providers totalled 208. Of these 69 were to the hospital physiotherapy service, mainly the community therapists, and 56 to private physiotherapy providers. A further 83 were principally social work referrals for home care via Accident Compensation Corporation or Support Works, or child concerns. In total 18 different pathways or primary care services received referrals.

\section{Allied Health Service Feedlback}

Consumers returned 51 questionnaires, a return rate of $60 \%$. Table 4 shows the feedback from the allied health consumers. Allied health consumers found the service helpful in providing information, treatment, practical support and giving follow up advice $(n=48)$. No concerns of not seeing the doctor were mentioned. Many consumers who had primary physiotherapy contacts appreciated the reduced waiting times (compared with what they were expecting), and found the service supportive and professional $(n=23)$. Other consumers appreciated the allied health follow up call or onward referrals to community services $(n=13)$, and being able to talk to someone empathetic $(n=8)$. There were no suggestions given for improvements in the service received.

\section{ED Staff Feedlback}

Questionnaires were returned from 42 ED staff, an $80 \%$ response rate. Table 4 shows how helpful they felt the service was to ED. Replies to open ended questions were collated and were found to be positive. Comprehensive quality patient care providing better outcomes (76\%) was what ED staff valued most from the allied health ED service. These staff appreciated the individual expertise the professions were able to offer ED (73\%), finding a social worker experienced in client psycho social needs of immense benefit (33\%) and facilitating multiple presentation reduction (7\%). Staff appreciated the physiotherapy musculoskeletal, mobility and falls risk assessments and management (35\%). Timely allied health interventions helped ED flow (57\%). The service was seen as an effective use of staffing resources, taking pressure off the medical and nursing team to let them concentrate on their roles of treating medically unwell ATS triage 1, 2 and 3 patients (38\%). Allied health staff also supported young doctors, providing a second opinion on assessment and management of presentations. As for care improvements and cost savings, improved safety of ED discharges rated highly (64\%), along with decreased admissions (38\%), and fewer representations (38\%). Increased community liaison and follow up (21\%) all added to a holistic client centred model of care (12\%).

Table 4: Consumer and Emergency Department staff feedback

\begin{tabular}{lccc}
\hline 1-5 scale of value & $\begin{array}{c}\text { Consumers } \\
\text { Allied Health } \\
(\mathrm{n}=51)\end{array}$ & $\begin{array}{c}\text { ED Staff } \\
\text { Physiotherapy } \\
(\mathrm{n}=42)\end{array}$ & $\begin{array}{c}\text { ED Staff } \\
\text { Social Work } \\
(\mathrm{n}=42)\end{array}$ \\
\hline 1 = Extremely helpful & 44 & 39 & 41 \\
2 = Very helpful & 6 & 3 & 1 \\
$3=$ Helpful & 1 & 0 & 0 \\
$4=$ Not very helpful & 0 & 0 & 0 \\
$5=$ Unhelpful & 0 & 0 & 0 \\
\hline
\end{tabular}

Notes: ED, Emergency Department 
Staff felt the impact of discontinuing the allied health in ED service would be huge, with an increased workload for medical and nursing staff and a return to coordinating multiple services (40\%). An increased length of stay was expected through having to page allied health staff stationed elsewhere in the hospital. They felt this would be a step backwards in service provision losing specialist input (38\%), with again the threat of unsafe discharges, admissions for social and mobility issues, and representations (26\%). Respondents considered allied health an integral part of a modern ED service, addressing social issues and improving staff morale and patient satisfaction (48\%). When asked for ideas on service improvement, extending allied health into after hours (26\%), weekend service (45\%) and allocating a designated office space (5\%) were mentioned.

ED staff reported that the allied health assessment and interventions of ED patients appeared to prevent an average of three hospital admissions per week; an estimated total of 66 prevented admissions over the initial 22 weeks.

\section{Stakeholder Feedback}

All stakeholders replied to the questionnaires, providing feedback as to how the pilot project had impacted on their diverse roles. Improved communication across the hospital and community had eventuated, through background work done to support patients admitted to hospital, and early identification of rehabilitation patients. Additional interventions meant fewer hospital admissions and positive links to primary providers. There was a change in workload with increased community physiotherapy referrals, decreased occupational therapy referrals for non-complex presentations, and acute allied health staff no longer being called to ED as well as having to manage their own inpatient workload.

Stakeholders felt the impact of allied health in ED on patients were: improved timely access for patients to ED, early assessment and identification of patient requirements, improved MDT interaction with higher quality information available, and that families were better supported.

Key stakeholders estimated financial benefits from the implementation of the pilot project over the first 22 weeks outweighed the costs. Cost savings were from an estimated 66 prevented admissions (average length of acute ward hospital stay 1-3 days). Furthermore there were 120 primary physiotherapy interventions where ED patients were assessed, treated and managed for less financial outlay than when using medical personnel. These cost savings outweighed the financial costs incurred with funding two full time allied health positions and administration outlay.

\section{DISCUSSION}

The pilot project embedding allied health in ED proved successful with 429 physiotherapy new patient contacts, 120 of these primary physiotherapy interventions and 320 social work new patient contacts over the first 22 weeks. Patients found the allied health input extremely helpful. Feedback from ED staff and key stakeholders was overwhelmingly positive with improved safety of discharges from ED to the community and comprehensive patient care rating highly important. Linking with primary health services helps improve care in the primary sector and keep patients out in the community where possible, a model of care better suited to manage long term conditions (MOH 2011). With allied health working as part of an interdisciplinary ED team to provide comprehensive patient care, approximately 66 admissions to hospital were estimated to have been averted. Other cost savings harder to quantify were reduced representations due to social or mobility issues and falls reduction due to screening and appropriate intervention.

Multiple presenter management plans help guide clinicians in any future ED presentations, and are regularly reviewed as required to respond to clients' changing needs. It was deemed helpful to have a social worker with the time and skills to support patients and families in stressful situations in hospital; this strength based approach is in keeping with the principles of Whānau Ora, a Ministry of Health initiative to place families at the centre of service delivery (MOH 2012)

This project had similar findings to other studies (Lau et al 2008, McLellan et al 2005, Morris et al 2015) in terms of positive feedback from patients, staff and stakeholders, and supports the benefit of a team approach in addressing the needs of complex presentations (Moss et al 2002). Where this study differs is in viewing both social work and physiotherapy as part of an allied health team, and also in their individual roles, providing data to support both professions being embedded within the ED service. This is particularly pertinent to social work service, which has limited data studies to date. In a small/medium sized hospital ED department this fluidity of practice best provides for individual patient needs and fills the gaps in service provision as required, making full use of staffing resources.

There were noticeable differences between social work and physiotherapy ED patient contacts. Physiotherapy had more new contacts, but fewer follow up interventions. This was largely due to the physiotherapy referral pathways from the ED service to other providers to continue appropriate care such as: (1) private physiotherapy and primary care providers, (2) community physiotherapy/occupational therapy, (3) community based services such as Falls Prevention, (4) fracture clinic, and (5) inpatient care. The social work ED service had fewer new referrals but a marked increase in follow up contacts, largely due to client psychosocial issues requiring further input, and community liaison requirements. The number of contacts increased (especially for physiotherapy) in the second data collection. This increase was due to reduced non-contact time taken up with pilot related activities and therefore increased amount of time available on the floor for patient contact. Referrals increased as ED staff became more aware of the benefits of allied health clinicians' interventions and developed a better understanding of what patient presentations could benefit and be referred. Also the physiotherapist's ability and confidence to assess and manage a wider range of injuries such as plaster skills for fracture management increased.

The time of service delivery (Figure 3) showed an increase in contacts mid-morning. The Allied Health After Hours folder captured patients requiring allied health input from the previous evening or weekend, which were actioned early the next working day. Also this time was often used for follow up contacts, mainly social work, which could account for the 
increase in contacts at this time. Trials of working later shifts into the evening appeared to have positive benefits for ED staff and patients, especially on busy days, but not enough to justify altered hours of working. Contact data showed benefits to ED of allied health working weekend shifts, particularly on Saturdays. Weekend shifts would have been appreciated by ED staff but were not funded.

There was resistance from some ED staff at first initiation of the pilot project, but this dissipated once the benefits of allied health input became obvious. The medical and nursing staff were able to perform their individual roles more effectively and no longer had to attempt to provide additional interventions outside their normal practice scope, stretching already busy service demands.

\section{Limitations}

Although allied health interventions were thought to have prevented admissions, backed by the clinical team and stakeholder feedback, it is difficult to produce hard evidence to prove this. This is largely due to the multi-factorial nature of complex presentations and variables in the ED environment. With an estimated three admissions a week averted it is not easy to show a trend, though each admission is highly significant, and has ongoing ramifications. Similar problems were encountered in a previous study (Moss et al 2002), which showed that hospital admissions were reduced post implementation but multiple variables could not be fully excluded.

Triage wait times have been used in other studies (Gill and Stella 2013, McClellan et al 2005, Morris et al 2015) to prove effectiveness of primary physiotherapy service in reducing waiting times. This would have been difficult to use in our study with physiotherapists treating both primary and secondary referrals, and with other co-jointly running initiatives, such as encouraging people with minor injuries to seek treatment in the primary care sector rather than ED. Again, reduced workload of medical and nursing staff is hard to quantify and subjective, though proof of contact data shows that interventions not only added to patient care but decreased patient numbers these disciplines would have needed to manage, so they could focus on higher triage medically unwell patients.

The allied health service was unable to deliver a 24 hour service, in line with the ED staffing structure. By ensuring an allied health service was present over much of the busiest time during the week, aided by the "Allied Health After Hours" referral folder to reach patients presenting out of normal working hours, the gap in service was minimised.

\section{CONCLUSION}

The benefits of allied health embedded in the emergency department were clearly evident after a six month pilot project of introducing full time positions of both social work and physiotherapy to Nelson Hospital Emergency Department, as shown in contact data and feedback surveys.

All initial aims of the pilot were fulfilled. The allied health team input provided a multi-faceted interdisciplinary team approach to patient care in a timely manner, facilitating safe discharge back into the community with linkages into primary care, preventing admissions to hospital where appropriate. Multiple presentations were reduced, with management plans in place. Primary physiotherapy contacts helped ED flow. Managing consumers' physical, mobility, rehabilitation, support and psychosocial needs and advocacy filled gaps in the traditional medical ED model, providing a more holistic client centred model of care.

Following the pilot full time physiotherapy and social work positions have been appointed and allied health has been permanently embedded in Nelson Emergency Department since December 2012, and has now been extended to the Wairau Hospital.

\section{KEY POINTS}

1. Allied health in ED work as part of an interdisciplinary team to facilitate timely and safe ED discharge and link patients with primary health services.

2. Allied health in ED assists in providing comprehensive client centred patient care.

3. Primary contact physiotherapy service has high patient satisfaction and aids ED flow.

\section{PERMISSIONS}

Permission has been granted by NMDHB management for figures and tables from the NMDHB 2012 pilot to be published in the New Zealand Journal of Physiotherapy by Hilary Exton, Director and Service Manager of Allied Health NMDHB and co author of this paper, who can be contacted via email if there are any queries.

\section{DISCLOSURES}

Financial support for the Pilot project came from the Nelson Marlborough District Health Board budget, covering staffing and administration costs for the 6 months of the pilot.

The authors of this paper have all been closely involved with the 2012 pilot and instigation of Allied Health in the Emergency Department. Care has been taken to objectively present all information, and there has been no compromise of the information this paper contains.

\section{ADDRESS FOR CORRESPONDENCE}

Clare Holmes, Physiotherapy Department, Nelson Hospital, Waimea Road, Nelson 7010, New Zealand. Telephone: 03 5393793. Email:clare.holmes@nmhs.govt.nz

\section{REFERENCES}

Australian and New Zealand Society for Geriatric Medicine (2008) The management of older patients in the emergency department. Position Statement No 14.

Bywaters P, McLeod E (2003) Social Care's Impact on Emergency Medicine: a model to test. Emergency Medicine Journal 20:134-137.

Canterbury District Health Board (2006) Front Door Physio or The Physiotherapist in ED, Final Audit Report.

Farrell S (2014) Can physiotherapists contribute to care in the emergency department? Australasian Medical Journal 7 (7): 315-317. 
Gill S, Stella J (2013) Implementation and performance evaluation of an emergency department primary practitioner physiotherapy service for patients with musculoskeletal conditions. Emergency Medicine Australasia 25 (6): 558-564.

Health Practitioners Competence Assurance Act (2003) Physiotherapy Board NZ. http://www.legislation.govt.nz/act/public/2003/0048/latest/whole.htm http://www.physioboard.org.nz/scopes-physiotherapy.

Jibuike O, Paul-Taylor G, Maulvi S, Richmond P, Fairclough J (2003) Management of soft tissue knee injuries in an accident and emergency department: the effect of the introduction of a physiotherapy practitioner. Emergency Medicine Journal 20: 37-39.

Lau P, Chow D, Pope M (2008) Early physiotherapy intervention in an Accident and Emergency Department reduces pain and improves satisfaction for patients with acute low back pain: a randomised trial. Australian Journal Physiotherapy. 54(4): 243-249.

McClellan C, Benger J (2005) A study into the impact of an extended scope physiotherapy practitioner on: waiting times, patient satisfaction and long-term outcomes from soft tissue ankle injuries in an emergency department. United Bristol Healthcare Clinical Research Report, Academic Department of Emergency Care, University Hospitals Bristol NHS FoundationTrust, Bristol.

McClellan C, Cramp F, Powell J, Benger J (2010) Extended scope physiotherapists in the emergency department: a literature review Physical Therapy Reviews 15 (2):106-111. doi 10.1179/174328810X1271900906 0344.

Ministry of Health NZ (2008) Recommendations to Improve Quality and the Measurement of Quality in New Zealand Emergency Departments. ISBN: 978-0-478-31897-5. http://www.health.govt.nz/system/files/documents/ publications/quality-ed-jan09.pdf.

Ministry of Health NZ (2011) Guidance for New Zealand Emergency Departments regarding the interface with primary health care. http:// www.health.govt.nz/our-work/hospitals-and-specialist-care/emergencydepartment/interface-primary-health-care.
Ministry of Health NZ (2011) Targeting Emergencies shorter stays in Emergency Departments. ISBN: 978-0-478-37442-1. http://www.health. govt.nz/system/files/documents/publications/targeting-emergencies-healthtarget.pdf.

Ministry of Health NZ (2011) Better sooner more convenient health care in the communityhttp://www.health.govt.nz/publication/better-sooner-moreconventient-health-care-community.

Ministry of Health NZ (2012) Whanau Ora programme (2012) http:// www.health.govt.nz/our-work/populations/maori-health/whanau-oraprogramme.

Morris J, Vine K, Grimmer K (2015) Evaluation of performance quality of an advanced scope physiotherapy role in a hospital emergency department. Patient Related Outcome Measures 2015:6 191-203.

Moss J, Flower C, Houghton L, Moss D, Nielsen D, Tayor D (2002) A multidisciplinary Care Coordination Team improves emergency department discharge planning practice. Medical Journal Australia 2002:177:427-431.

New South Wales Health $(2006,2012)$ Emergency Department Models of Care http://www.health.nsw.gov.au/Performance/publications/ed-modelof-care-2012.pdf.

The National Ageing Research Institute, Australia (July 2007) Falls Prevention Guidelines for the Emergency Department. The Australian Government Department of Health and Aging.

Rea H, Kenealy T, Horwood F, Sheridan N, Parsons M, Wemekamp B, Winter F, Maingay G, Degeling P (2010) Integrated systems to improve care for very high intensity users of hospital emergency department and for long-term conditions in the community New Zealand Medical Journal 123:1320:76-85 ISSN11758716.

Van Pelt J (2010) Making Caring Connections, Cutting Costs - Social Work in the Emergency Department Social Work Today 10(6): 12 